\title{
Der Staat im Staat
}

\section{Rainer M. Kaelin}

Dr. med., Facharzt für Pneumologie und Innere Medizin, vormals Vizepräsident der Lungenliga Schweiz und der Lungenliga Waadt, Vizepräsident von OxyRomandie/OxySuisse; Mitglied der FMH

\section{Die Instrumentalisierung der Schweiz durch den Zigarettenhersteller Philip Morris} (PM), der sich das Ansehen eines Hauptsponsors des Schweizer Pavillons an der Weltausstellung in Dubai 2020 gesichert hatte, wirft die Frage auf, inwiefern in unserem Lande die politischen Entscheidungsträger die Rahmenbedingungen der Wirtschaft festlegen oder ob nicht eher diese die Politik bestimmt.

An der Uni Lausanne wurde die erste Beschreibung der Beziehungen von Zigarettenfabrikanten zu Wissenschaft und Politik der Schweiz als Doktorarbeit in Medizin-Biologie eingereicht [1]. Mit dem Tabakproduktegesetz (TabPG) sind diese in den Fokus gerückt. Sie erklären die verhinderten Werbeverbote, die Ablehnung der Zwillingsinitiative 1992, die Mängel des Passivrauchgesetzes und die bisherige Weigerung des Parlaments, die Tabakrahmenkonvention der WHO zu ratifizieren und umzusetzen.

\section{Interessengruppen als parastaatliche Strukturen}

Interessengruppen der Wirtschaft organisierten sich früh im liberalen Bundesstaat. Sie waren und sind bedeutend, weil der Bund über begrenzte Mittel verfügt. Der Einfluss der Interessengruppen nahm mit ihrer landesweiten Präsenz zu; sie werden daher auch "parastaatliche Strukturen" genannt [2]. Die ASFC (Association Suisse des fabricants de Cigarettes, später CISC, Communauté des industriels Suisses des Cigarettes, seit 2004 "Swiss Cigarette») konstituierte sich 1933. Ihre Leitung war von 1936-2004 vier Nationalräten anvertraut, die ihr Präsidium nach der Wahl antraten. Die Bedeutung, welche die ASFC dieser Vertretung zumass, zeigte sich, als Nationalrat Fernand Corbat, dem die Wiederwahl in den Nationalrat 1979 misslang, deswegen als Präsident der Zigarettenhersteller zurücktreten musste [3]. Denn mit dem Parlamentssitz stehen Einflusskanäle zur Verfügung: Kommissionen, Anhörung bei Vernehmlassungen, Kontakte zur Verwaltung, Selbstregulierung, Einfluss auf andere Parlamentarier, Medien und öffentliche Meinung.

In der Schweiz ist die «Präparlamentarische Phase» der Gesetzgebung entscheidend, da Kompromisse in Kommissionen ausgehandelt werden, lange bevor die Gesetzesentwürfe im Parlament debattiert werden [4].
Finanzstarken Interessengruppen wie Swiss Cigarette, umfassend die multinationalen Firmen PM, British American Tobacco (BAT) und Japan Tobacco international (JTI), stehen als Druckmittel zudem das Referendum zur Verfügung.

Die Grenzen extraparlamentarischer Kommissionen, Kompromisse auszuhandeln, zeigten sich anschaulich anhand der Tabakkommission (1987-1996), die durch Bundesrätin Ruth Dreifuss aufgelöst wurde, weil kein Konsens mehr möglich war. Dies geschah, weil die ASFC, am Bundesamt für Gesundheit vorbei, eine «ausgewogene, unvoreingenommene Zusammensetzung der Kommission» erreichte, d.h. Einsitz in die Kommission erhielt für «je einen Vertreter der Zigarettenindustrie, der Zigarren- und Pfeifentabakindustrie, der Tabakpflanzer, der Tabakhändler, und zwei Vertreter der Werbeindustrie» [5].

\section{Selbstregulierung: Jugendschutz durch Lauterkeitskommission?}

Interessengruppen bevorzugen Selbstregulierung. Ohne Gesetze erlaubt diese, Lösungen einzuführen und so die Politisierung des Problems zu vermeiden, was sonst die Interessenkonflikte offenlegen würde. Selbstregulierung wird als Beweis vorgezeigt, Gesetze seien unnötig, und man stellt sich auch als verantwortliches Element der Gesellschaft hin. So hat sich seit 1955 die Tabakindustrie sechs Mal selbst Regeln zur Werbung auferlegt oder diese überarbeitet, zuletzt 2005 mit der Vereinbarung zwischen Swiss Cigarette und Lauterkeitskommission. 1992 geschah dies unter dem Druck der Zwillingsinitiative (Verbot von Tabak- und Alkoholwerbung). Der Vizepräsident von PM dazu: «Der Zweck des Codes ist ein verstärkter Jugendschutz, angesichts der Parlamentsdebatte zur Zwillingsinitiative. Die Absicht der Industrie und ihrer Alliierten ist, 1. die Parlamentarier zu überzeugen, dass ein Gegen- 
vorschlag unnötig ist und dass Jugendschutz besser durch Selbstregulierung geschieht ... und 2. die Stimmbürger zu bewegen, die Initiative abzulehnen, wegen ihrer Zwecklosigkeit. Um höchste Glaubwürdigkeit zu erreichen, wird der Code gegengezeichnet durch 'Publicité Suisse' und ihre Durchführung wird überwacht durch die Lauterkeitskommission» [6]. Mit der Ablehnung der Initiative segnete das Parlament die Selbstkontrolle ab. Diese gab sich offiziellen Status, indem sie Politiker einsetzte und so Zigarettenhersteller, Politik und Werbefachleute vernetzte. Die Regelung der vom Werbefachverband bestellten Lauterkeitskommission wurde von Doris Leuthart, ihrer damaligen Präsidentin und Präsidentin der CVP, unterzeichnet. Präsident der Werbefachleute war seit 2007 Ständerat Carlo Schmid, vormals Präsident der CVP, der 2016 von Ständerat Filippo Lombardi, Fraktionschef der CVP, abgelöst wurde. Aktuelle Präsidentin der Lauterkeitskommission ist Nationalrätin Christine Bullard-Marbach (CVP). Obwohl sich die Lauterkeitskommission als unabhängig bezeichnet [7], kann sie das nicht sein: Im Gegensatz zu denen anderer Bereiche hat eine von ihr festgestellte durch die Tabakindustrie begangene Übertretung keine Sanktionen zur Folge, sondern wird lediglich weitergeleitet an ... Swiss Cigarette [8]. Die Interessenkonvergenz zwischen Werbesektor und der Tabakindustrie spiegelt sich auch in der Struktur des Werbeverbandes "Kommunikation Schweiz» wider [9]. Die Tabakindustrie ist hierin vertreten als Auftraggeber von PR-Aktionen, als Urheber von Produktewerbung, als Beurteiler des Werbesektors. Schutz der Jugend vor Werbung ist kein Anliegen der Lauterkeitskommission. Dies ersieht man aus der mit Stichentscheid der Präsidentin abgelehnten Klage des Verbandes der Lehrer/innen der Schweiz [10]. Im Gegensatz zu diesen fand die Lauterkeitskommission nichts zu bemängeln an einer Werbeaktion von Mediamarkt, die Kinder für ihre schulischen Leistungen mit Geld belohnte, um sich in diesem Bevölkerungssegment populär zu machen [10].

Auch die Verwaltung stützt die Selbstkontrolle. Als im Dezember 2018 die E-Zigarette JUUL in der Schweiz eine Epidemie von Nikotinsüchtigen auslöste, da ihr Verkauf behördlich nicht eingeschränkt wurde, rief der Bundesrat die E-Zigaretten-Händler zu einem runden Tisch zusammen. Das Resultat war ein «Codex», der einer PRAktion gleicht: Die Partner der Swiss Vape association (www.svta.ch/kodex) verpflichten sich freiwillig, keine E-Zigaretten an Minderjährige zu verkaufen und an diese keine Werbung zu richten. Sie versprachen, dies durchzusetzen, aber weder Sanktionen noch eine beurteilende Instanz wurden genannt. Von Promotionsund Sponsoringverboten war keine Rede.

\section{Vernetzungen Industrie-Politik}

Informatives Gästebuch der Firma Burrus: Bundespräsident Honegger 1982, Wirtschaftsminister Delamuraz 1988, Vertreter von COOP und Denner, der Banken, Ringier und Co, Tages-Anzeiger und andere bedanken sich für freundschaftlichen Empfang. Archivdokumente von PM belegen 1982 Abmachungen mit den Regierungsräten J. Cavadini und J. Béguin [11] in Neuenburg. In ihrem «Corporate Affairs Plan 1987» liest man: «Mehr Mitglieder rekrutieren für den 21-köpfigen 'Tabakparteiausschuss' und diese anregen, Industrieansichten überzeugend dem Parlament zu kommunizieren. Diese Alliierten in das Frühwarnsystem integrieren» [12].

Geld unterhält Beziehungen: «Wir müssen daher um eine bedeutend höhere Beteiligung ihrerseits bitten" [13]. Der Brief der Liberalen Partei der Waadt belegt, dass die Parteien von Geldern der Wirtschaft abhängen. Olivier [1] zeigt, dass PM von 1992 (Jahr vor der Abstimmung über die Zwillingsinitiative) bis 1995 durchschnittlich 50000 CHF pro Jahr an die bürgerlichen Parteien spendete, gut investiertes Geld.

Eine Studie der Firma R.J. Reynolds (später JTI) zur Ablehnung der Zwillingsinitiative weist auf die Massnahmen der CISC hin: «Starkes Lobbying: Regierung, Parlament, politische Parteien, Wirtschaft, Sport, Kultur» waren, zusammen mit dem politischen System und der Pro-Tabak-Koalition entscheidend [14]. Einige Wochen nach ihrem Erfolg trafen sich CISC und die Parteien CVP, SVP, Freisinnige und Liberale, um «sich für ihren Einsatz in der Abstimmungskampagne gegen die Zwillingsinitiative» zu bedanken. Die Präsidenten Carlo Schmid, Franz Steinegger (Freisinnige) und Hans Uhlmann (SVP) waren zugegen sowie die Parteisekretäre Raymond Loretan (CVP) und Philippe Boillod (Liberale). Das Protokoll hält fest: «die Mitglieder der Freisinnigen schätzten die ihnen vorgetragene Vorstellung der Schweizer Tabakindustrie, denn sie wussten gar nicht, dass diese Industrie auch eine Exportbranche ist. Die Partei schätzt diese Art Kontakt und ist bereit, diese fortzusetzen.» - «Zum Schluss bietet der Präsident der Volkspartei der Industrie die Möglichkeit, sich an die Partei zu wenden, falls Probleme zusammen gelöst werden sollten.» - «Die liberale Partei hält sich gerne zu Verfügung unserer Industrie, um zur Lösung eventueller Probleme behilflich zu sein» - «... die Industrie (organisiert) zweimal jährlich Zusammenkünfte mit Parlamentariern, die der Tabakbranche nahestehen.»

\section{Tabakprävention im Milizparlament des föderalistischen Kleinstaates}

Von Politikern des Milizparlamentes werden Interessenkonflikte häufig verdrängt; ihr persönliches Loyali- 
tätsverhalten lässt ihnen nicht bewusst werden, dass man sie instrumentalisiert. So überwiegen in ihrer liberalen Rhetorik zu «libertiziden Regeln» die PRParolen der Industrie. Sie vergessen, dass im aufgeklärten Liberalismus die Freiheit der einen (Tabakindustrie) nicht zum Schaden anderer gereichen darf. So heisst es 1979 (Industriedokument): «Die Initiative akzeptieren, bedeutet dem Bund unsere Vormundschaft anvertrauen. (Sie) verletzt nicht nur die Handels- und Gewerbefreiheit, sondern vorallem unsere individuelle Freiheit» [15]. Analog heisst es 2012 zur Initiative zum Schutz vor Passivrauch: «Ein absolutes Rauchverbot wäre eine massive Verletzung der Privatsphäre. Die Initianten wollen die Bürgerinnen und Bürger bevormunden» [16]. Das Rauchverbot im geschlossenen öffentlichen Raum wird zum «absoluten» Rauchverbot deformiert. Neben Freiheit wird Föderalismus zum schützenswerten Prinzip. So meint das «Komitee gegen das absolute Rauchverbot»: «Diese Initiative widerspricht dem Foederalismus, indem sie den Kantonen eine strikte Regel aufzwingt. Es ist daher nötig, die Initiative abzulehnen» [16]. Die «liberale» Botschaft wird über die Allianz der Wirtschaft für eine massvolle Prävention (AWMP) und durch Kommunikation Schweiz verbreitet. Ihre Geschäftsführerin meint zum Tabakwerbeverbot zum Schutz von Jugendlichen und Kindern 2018: «Die Selbstregulierung genügt, zusammen mit dem griffigen Tabakproduktegesetz vollauf. Was darüber hinausgeht, schränkt nicht nur die Wirtschafts- und Werbefreiheit ein. Noch schlimmer ist, dass den Schweizer Bürgerinnen und Bürgern mit einem Totalverbot die Mündigkeit abgesprochen wird» [17]. Damit blendet sie aus, dass Nikotinprodukte nicht Lebensmittel sind, sondern abhängig machen, weshalb deren Werbung besonders Kinder gefährdet, eigentliches Zielpublikum, das die Initiative schützen will.

Dr. med. Rainer M. Kaelin

Plantay 53

CH-1163 Etoy VD

palmier.kaelin[at]bluewin.ch ist naheliegend, damit auch die Verzögerungstaktik im Werdegang des TabPG zu erklären, das nun, gemäss Ständerat Werbeverbote enthalten soll, nachdem dieselben Parlamentarier sie im ersten Entwurf zurückgewiesen hatten. Dies, wie auch die anderen Alibi-Präventionsmassnahmen, beschlossen Industrie-freundliche Volksvertreter, die den grundlegenden Interessenkonflikt zwischen öffentlicher Gesundheit und Industrie ignorierten und die Tatsache, dass Jugendschutz ohne umfassendes Werbeverbot nicht möglich ist. Die Vernetzungen erklären, warum die Verpflichtungen der FCTC im Schweizer Parlament erst 15 Jahre nach deren Implementierung in andern Ländern zur Sprache kommen.

Interessenkonflikte sind im Milizparlament unvermeidlich. Falls nicht politischer Wille und gesetzlicher Zwang zur Offenlegung der Beziehungen zwischen Industrie und Politkern sowie der Finanzflüsse zu den Parteien bestehen, unterminieren diese den demokratischen Prozess, da sie das Vertrauen der Bevölkerung in den Staat untergraben.

\section{Literatur}

1 Olivier J. Les fabricants de cigarettes face à la question tabac et santé en Suisse (1962-2003). Thèse de doctorat MD-PhD, Biologie et médecine, UNIL 2019. Als PDF abrufbar, www.serval.unil.ch

2 Eichenberger P, Mach A. Organized capital and coordinated market economy. Swiss business interest associations between socio-economic regulation and political influence. In: Mach A, Trampusch C (dir): Switzerland in Europe. Continuity and change in the Swiss political economy. London, New York: Routledge; 2011, p. 71.

3 Eggly JS. Manque d'humanité et faute politique. M. Corbat quitte l'industrie Suisse du Tabac, Journal de Geneve, $1^{\text {er }}$ avril 1980.

4 Gava R, Varone F, Mach A, Eichenberger S, Christe J, Choa-Blanco C. «Interest groups in Parliament: Exploring MP's interest affiliations (2000-2011)». Swiss Political Science Review. 2017;23(1):81.

5 British American Tobacco Records. «Federal Commission on problems associated with tobacco", 22. März 1988. Zitat in Olivier J, (1), S. 100-1.

6 Diserens G. Weekly Highlights: week ending June 19, 1992, Switzerland-Finland/Scandinavia-Baltics Duty free. 19. June 1992, pp. 1-2. Philip Morris Records. Zitat in Olivier J, (1), S. 102-3. https:// www.industrydocumentslibrary.ucsf.edu/tobacco/doc/zgkc0118

7 https://www.faire-werbung.ch

8 Kaelin RM. Jugendschutz ohne Werbeverbot? Schweiz Ärzteztg. 2017;98(41):1347-9.

9 https://ks-cs.ch

10 www.lch.ch: SER (Syndicat Enseignants Romands) bedauert Entscheid der Lauterkeitskommission (besucht am 31.7.2019).

11 Pantet R. Note sur la séance du 16 novembre 1982 avec MM Cavadini et Béguin, Conseillers d'Etat, p. 1, Philip Morris Records, clb56e00. Zitat in Olivier J, (1), S. 112-3.

12 «Corporate affairs plans», 25 November 1987, p. 6, Philip Morris Records, ity32e00. Zitat in Olivier J, (1), S. 113.

13 Gamboni PV, Albisser G, Rham W. Lettre du parti libéral vaudois à Philip Morris Europe SA, 19 février 1982.

14 "Swiss advertising ban case study», November 28, 1993, p. 19. R.J. Reynolds, oim33d00. Zitat in Olivier J, (1), S. 93.

15 Fankhauser W. «Good Young Templars' Motion», 23 February 1979. British American Tobacco Records.

16 Arreté fédéral concernant l' initiative populaire Protection contre le tabagisme passif: www.parlament.ch/centers/documents/ fr/11-025-argumentarien-contra-f.pdf

17 Gamper U. Tabakwerbeverbot zum Schutz von Kindern und Jugendlichen? - Dagegen, Zeitlupe 11/2018. 